\section{Determinantes da mortalidade neonatal a partir de uma coorte de nascidos vivos, Montes Claros, Minas Gerais, 1997-1999}

\author{
Determinants of neonatal mortality \\ in a cohort of born alive infants, \\ Montes Claros, Minas Gerais, 1997-1999
}

Eunice Francisca Martins 1

Gustavo Velásquez-Meléndez 1

1 Departamento Materno-Infantil e Saúde Pública Escola de Enfermagem. Universidade Federal de Minas Gerais. Av. Alfredo Balena, 190. Belo Horizonte, MG, Brasil. CEP: $30.130-100$

\begin{abstract}
Objetives: to identify the factors of risk for the neonatal mortality with information from born alive information system and mortality information system in city of Montes Claros, in the period of 1997 through 1999.

Methods: the linkage technique has been used to link the declarations of death with the respective declarations of the infants who were born alive. The identification of factors associated to neonatal mortality has been done through bivaried and multivariable analysis, obtaining the calculus of risk and its $95 \%$ confidence interval.

Results: the infants who were born alive were 20.506 and the deaths 275 which resulted in a coefficient of neonatal mortality of 13.4 to one thousand who were born alive. Through the multi-varied analysis, independent factors of risk were obtained for the occurrence of neonatal deaths to prematurely born infants, the low weight when they are born and the score of Apgar lower than seven in the first and fifth minute of life.

Conclusions: efforts have been made to improve the obstetrics and neonatal assistance in the city of Montes Claros, providing conditions for safe pregnancy and birth, thus increasing the chances of survival in the beginning of life.
\end{abstract}

Key words Infant mortality, Information systems, Epidemiologic surveillance

\section{Resumo}

Objetivos: identificar os fatores de risco para a mortalidade neonatal a partir das informações contidas no Sistema de Informação de Nascidos Vivos e Sistema de Informações de Mortalidade na cidade de Montes Claros, no período de 1997 a 1999.

Métodos: foi utilizada a técnica de linkage para concatenar as declarações de óbitos com as respectivas declarações de nascidos vivos. A identificação dos fatores associados à mortalidade neonatal foi realizada através das análises univariada e multivariada; obteve-se o cálculo dos riscos e seus intervalos de confiança de $95 \%$.

Resultados: os nascidos vivos foram 20.506 e os óbitos 275, resultando um coeficiente de mortalidade neonatal anual médio de 13,4 por mil nascidos vivos. Através da análise multivariada constituíram-se fatores de risco independentes para a ocorrência dos óbitos neonatais a prematuridade, o baixo peso ao nascer e o escore de Apgar inferior a 7 no $1^{\circ}$ e $5^{o}$ minutos de vida.

Conclusões: esforços devem ser dirigidos no sentido de garantir uma assistência obstétrica $e$ neonatal na cidade de Montes Claros que propicie condições para uma gestação e nascimento seguros, favorecendo, assim, a sobrevivência no início da vida. Palavras-chave Mortalidade infantil, Sistemas de informação, Vigilância epidemiológica 


\section{Introdução}

A taxa de mortalidade neonatal, proporção de óbitos ocorridos nos primeiros 27 dias de vida, é um indicador negativo de saúde que, no Brasil, apresenta níveis elevados não compatíveis com o potencial econômico e tecnológico, visto que na maioria das circunstâncias esse evento é considerado evitável através da utilização de tecnologias atualmente disponíveis. ${ }^{1}$

No Brasil, a partir da década de 90, os óbitos neonatais passaram a ser o principal componente da mortalidade no primeiro ano de vida, em função das intervenções globais que favoreceram o decréscimo da mortalidade infantil pós-neonatal e da maior complexidade dos fatores biológicos, socioeconômi$\cos$ e assistenciais determinantes da mortalidade nos primeiros dias de vida. ${ }^{2}$

A redução da mortalidade neonatal é um grande desafio para os serviços de saúde, governos e sociedade, pelas altas taxas vigentes, concentradas nas regiões e populações mais pobres. Essa situação reflete as desigualdades sociais do país e a dificuldade de acesso, em tempo oportuno, aos serviços de saúde resolutivos e qualificados. ${ }^{3}$ Para se obter em resultados mais efetivos na redução desse componente da mortalidade infantil, há a necessidade de intervenção nos seus múltiplos fatores relacionados e de interação entre as ações de atenção à saúde, serviços de educação e assistência social para o atendimento às necessidades básicas capazes de garantir uma gestação e nascimento em condições que favoreçam uma sobrevivência segura. 4

A mortalidade infantil é avaliada pelas mortes ocorridas no primeiro ano de vida, sendo constituída pelos componentes neonatal (menores de 28 dias de vida) e pós-neonatal ( $29^{\circ}$ dia em diante). A mortalidade neonatal é dividida em precoce (menores de sete dias de vida) e tardia (do sétimo ao $28^{\circ}$ dias). $\mathrm{O}$ coeficiente de mortalidade neonatal é a razão entre o número de óbitos de crianças menores de 28 dias num determinado período, sobre o número total de nascidos vivos no mesmo período, multiplicado por 1.000 .5

Os fatores causais relacionados aos componentes neonatal e pós-neonatal da mortalidade infantil são diferentes. Nas primeiras semanas de vida os agravos decorrentes das condições da gestação e nascimento predominam como causas da mortalidade. Após esse período, a maior interferência se dá pelas doenças diarréicas, respiratórias e imunopreveníveis. ${ }^{6}$

Os determinantes da mortalidade neonatal são múltiplos e complexos, relacionando-se à interação de variáveis biológicas, assistenciais e socioeconômicas. As variáveis biológicas referem-se à mãe e ao recém-nascido e são as causas diretas dos óbitos neonatais. $\mathrm{O}$ acesso aos serviços de saúde e a qualidade da assistência prestada no pré-natal, sala de parto e os cuidados pós-natais ao recém-nascido são variáveis assistenciais capazes de interferir nos fatores de risco biológicos e socioecômicos para a mortalidade neonatal. As variáveis socioeconômicas indicam as condições em que vive a mãe, as quais são capazes de influenciar alguns efeitos das variáveis biológicas e dificultar o acesso a uma adequada assistência no período da gestação e nascimento. 7

A Figura 1 descreve um modelo explicativo hierárquico sobre os principais fatores relacionados à mortalidade neonatal à partir da revisão de estudos sobre o tema. ${ }^{7-12}$

Este estudo teve como objetivo identificar os fatores de risco para a mortalidade neonatal a partir de uma coorte de nascidos vivos filhos de mães residentes no município de Montes Claros, no período de 1997 a 1999. Espera-se que os resultados forneçam subsídios para a avaliação da situação da mortalidade neonatal e identificação de pontos estratégicos para intervenções, contribuindo para a redução das situações indesejadas de sofrimento, morbidade e morte que se contrapõem à alegria esperada do nascimento. 


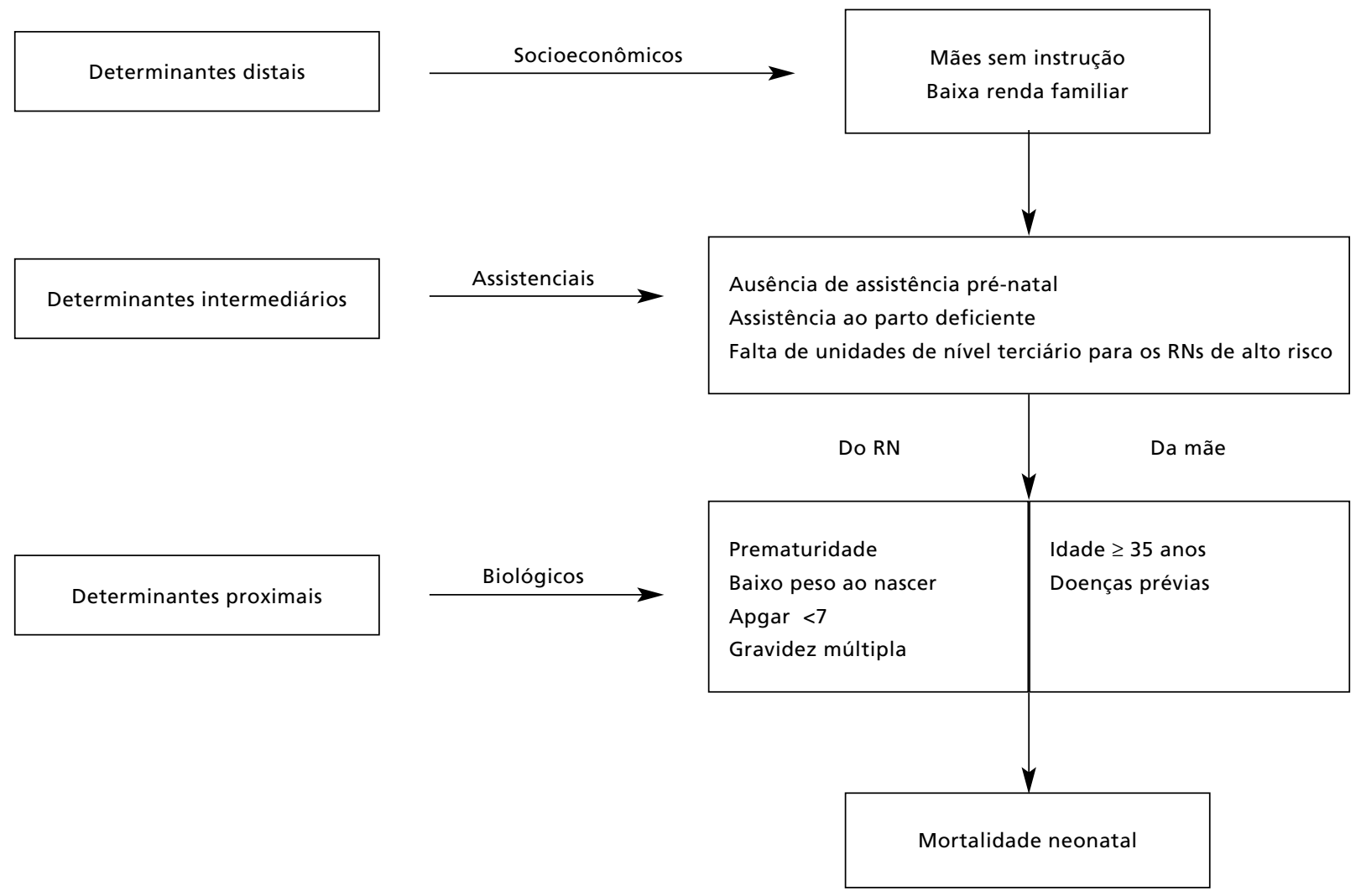

\section{Métodos}

Estudo do tipo coorte. A população constituiu-se de 20.506 nascidos vivos no período do 1 de janeiro de 1997 a 31 de dezembro de 1999, filhos de mães residentes no município de Montes Claros, MG, Brasil, e de 275 óbitos neonatais, 200 concatenados a essa coorte e 75 não concatenados por falta de dados da época do nascimento.

Os dados estudados foram provenientes do Sistema de Informação de Mortalidade (SIM) e do Sistema de Informação de Nascidos Vivos (SINASC), cedidos pela Secretaria de Saúde de Montes Claros. Foi utilizado o procedimento de linkage entre os bancos de dados de óbitos e nascimentos para identificar os nascidos vivos que evoluíram para o óbito no período neonatal e caracterizar esses óbitos segundo variáveis constantes na Declaração de Nascidos Vivos (DN). Através de um programa informatizado e de busca ativa nas maternidades conseguiu-se a linkage de 200 casos de óbitos às suas respectivas Declaração de Nascidos Vivos (DN).

O procedimento de linkage dos bancos de dados de nascimentos e óbitos neste estudo foi dificultado pela divergência do nome da mãe na Declaração de nascidos Vivos e na Declaração de Óbito, requerendo assim verificação de outros campos tais como sexo, idade e data de nascimento para confirmar a concatenação das duas declarações e também pelo não preenchimento da DN em alguns casos de morte prematura (primeiras horas ou dias de vida).

A variável dependente do estudo foi a ocorrência de morte nos primeiros 27 dias de vida e as independentes estão relacionadas ao recém-nascido (sexo, peso ao nascer, escore de Apgar no primeiro e quinto minutos de vida e idade gestacional), à gestação e parto (tipo de gravidez e parto, número de consultas de pré-natal e local do nascimento) e à mãe (grau de instrução, idade, filhos e abortos tidos).

As análises estatísticas foram realizadas utilizan- 
do-se os softwares Epi-info versão 6.0 e SPSS/PC, versão 8.0. Para testar a associação dos vários fatores com a mortalidade neonatal foram realizadas análises bivariadas e multivariadas utilizando a técnica da regressão logística. O ajuste para as variáveis de confusão no modelo de regressão foi realizado pela estratégia "passo a passo". Como medidas de força de associações foram utilizados o risco relativo e o odds ratio e seus intervalos de confiança em nível de 95\% (IC95\%). Todas as associações foram consideradas estatisticamente significantes em nível de $p \leq 0,05$. Foram desconsiderados todos os registros das DNs que possuíam pelo menos uma variável com valor omisso. Com esse procedimento, perderam-se 1491 registros, sendo a análise realizada de um total de 19.015 casos.

\section{Resultados}

O coeficiente de mortalidade neonatal $(\mathrm{CMN})$ para o município, no período estudado, foi de 13,4 por mil nascidos vivos.

A Tabela 1 apresenta o CMN para cada variável estudada e os resultados da análise bivariada para o óbito neonatal. O maior coeficiente de mortalidade neonatal por mil nascidos vivos foi 350 , encontrado entre os recém-nascidos que no quinto minuto de vida apresentaram escore de Apgar inferior a sete e o menor coeficiente foi três para as crianças nascidas de peso $\geq 2500$ g e para as nascidas a termo.

\section{Tabela 1}

Coeficiente de mortalidade neonatal (CMN) por 1000 nascidos vivos e Risco Relativo para óbito neonatal segundo variáveis da Declaração de Nascido Vivo. Montes Claros, MG, 1997 a 1999.

\begin{tabular}{|c|c|c|c|c|}
\hline Variáveis & CMN & RR (não ajustado) & IC95\% & p \\
\hline \multicolumn{5}{|l|}{ Sexo } \\
\hline Feminino & 11,7 & 1,0 & & \\
\hline Masculino & 15,0 & 1,2 & $1,01-1,62$ & 0,039 \\
\hline \multicolumn{5}{|l|}{ Peso } \\
\hline$\geq 2500 \mathrm{~g}$ & 3,0 & 1,0 & & \\
\hline$<2500 \mathrm{~g}$ & 77,0 & 29,48 & $21,38-0,66$ & $<0,001$ \\
\hline \multicolumn{5}{|l|}{ Apgar 1' } \\
\hline$\geq 7$ & 4,0 & 1,0 & & \\
\hline$<7$ & 102,2 & 25,7 & $19,24-34,39$ & $<0,001$ \\
\hline \multicolumn{5}{|l|}{ Apgar 5' } \\
\hline$\geq 7$ & 5,0 & 1,0 & & \\
\hline$<7$ & 350,0 & 54,18 & $41,59-70,57$ & $<0,001$ \\
\hline \multicolumn{5}{|l|}{ Idade gestacional } \\
\hline $37-41 s$ & 3,0 & 1,0 & & \\
\hline$<37 \mathrm{~s}$ & 147,0 & 48,47 & $35,63-65,92$ & $<0,001$ \\
\hline$\geq 42 \mathrm{~s}$ & 18,0 & 6,12 & $1,93-19,41$ & $<0,001$ \\
\hline \multicolumn{5}{|l|}{ Tipo de gravidez } \\
\hline Única & 9,0 & 1,0 & & \\
\hline Múltipla & 52,15 & 5,95 & $3,90-9,10$ & $<0,001$ \\
\hline \multicolumn{5}{|l|}{ Tipo de parto } \\
\hline Normal & 10,0 & & & \\
\hline Operatório & 11,0 & 1,11 & $0,83-1,49$ & 0,482 \\
\hline \multicolumn{5}{|l|}{ Consultas de pré-natal } \\
\hline Mais de seis & 6,0 & 1,0 & & \\
\hline Até seis & 9,0 & 1,54 & $1,08-2,21$ & 0,170 \\
\hline Nenhuma & 41,0 & 7,48 & $4,41-12,68$ & $<0,001$ \\
\hline \multicolumn{5}{|l|}{ Grau de instrução } \\
\hline Superior & 4,0 & 1,0 & & \\
\hline Nenhuma & 21,0 & 5,86 & $1,62-21,21$ & 0,002 \\
\hline Primeiro grau incompleto & 8,0 & 2,36 & $0,74-7,45$ & 0,131 \\
\hline Primeiro grau & 10,0 & 2,94 & $0,91-9,54$ & 0,058 \\
\hline Segundo grau & 7,0 & 2,0 & $0,61-6,54$ & 0,243 \\
\hline \multicolumn{5}{|l|}{ Idade materna } \\
\hline 20-34 anos & 9.0 & 1,0 & & \\
\hline$<20$ anos & 10,0 & 1,03 & $0,73-1,45$ & 0,863 \\
\hline$\geq 35$ anos & 12,0 & 1,32 & $0,80-2,18$ & 0,276 \\
\hline
\end{tabular}


As variáveis independentes que se associaram aos óbitos neonatais foram o baixo peso ao nascer (BPN); Apgar inferior a sétimo no primeiro e quinto minutos de vida; idade gestacional inferior a 37 semanas e superior a 42 semanas; nenhuma realização de consultas de pré-natais e nenhuma instrução materna.

Em relação à história obstétrica prévia, predominaram as mães primíparas sem relato de filhos mortos ou abortos anteriores e essas características não constituíram fator de risco para o óbito neonatal. A totalidade dos nascimentos que evoluíram para o óbito neonatal foi hospitalar e a maternidade do nascimento não se associou ao risco de morte neonatal.

$\mathrm{Na}$ análise multivariada, constituíram-se em fatores de risco independentes para o óbito no período neonatal o baixo peso ao nascer, a prematuridade e o escore de Apgar inferior a sete no primeiro e quinto minutos de vida. (Tabela 2)

Resultados da análise multivariada da mortalidade neonatal. Montes Claros, MG, 1997 a 1999.

\begin{tabular}{|c|c|c|c|}
\hline Variáveis & Odds Ratio & IC95\% & $\mathbf{p}$ \\
\hline $\begin{array}{l}\text { Peso ao nascer } \\
\qquad(1=\text { baixo peso; } 0=\text { peso normal })\end{array}$ & 4,94 & $2,91-8,38$ & $<0,001$ \\
\hline $\begin{array}{l}\text { Idade gestacional } \\
\qquad(1=\leq 37 \text { sem.; } 0=\geq 37 \text { sem } .)\end{array}$ & 5,68 & $3,39-9,52$ & $<0,001$ \\
\hline $\begin{array}{l}\text { Apgar } 1^{\circ} \text { minuto } \\
\quad(0-10)\end{array}$ & 0,75 & $0,66-0,86$ & $<0,001$ \\
\hline $\begin{array}{l}\text { Apgar } 5^{\circ} \text { minuto } \\
(0-10)\end{array}$ & 0,76 & $0,67-0,87$ & $<0,001$ \\
\hline
\end{tabular}

\section{Discussão}

A utilização dos sistemas de informações de nascidos vivos e de óbitos para identificar os determinantes da mortalidade neonatal foi viável, apesar de dificuldades na concatenação dos bancos de dados. Falhas no preenchimento da DO e DN foram também encontradas em outros estudos, 13,14 os quais sugerem para o aperfeiçoamento da qualidade do preenchimento desses documentos a capacitação profissional, a busca ativa nos órgãos de origem com solicitação de reparação dos dados incompletos e utilização destas informações na elaboração de políticas públicas de saúde.

O coeficiente de mortalidade neonatal de 13,4 por mil nascidos vivos $(\mathrm{NV})$ encontrado no período estudado na cidade de Montes Claros foi inferior aos dados nacionais e do estado de Minas Gerais que tiveram em média 20 e 17 óbitos a cada mil NV, respectivamente. 15 Entretanto é considerado alarmante quando comparado às taxas dos países desen- volvidos, nos quais ocorrem $10 \%$ dos nascimentos e $2 \%$ da mortalidade neonatal de todo o mundo. 16

O sexo não se associou à mortalidade neonatal na análise multivariada, concordando com alguns estudos, $9,12,17$ e discordando de outros 10 onde houve uma maior ocorrência de óbitos entre os recémnascidos do sexo masculino. A associação entre gravidez múltipla e mortalidade neonatal neste estudo foi esvaziada na análise multivariada, provavelmente em virtude do fato de a idade gestacional e o baixo peso ao nascer, muito prevalente entre os gemelares, serem variáveis independentes mais fortemente associadas à mortalidade. Resultados semelhantes foram evidenciados em estudos do mesmo gênero. 9,17

$\mathrm{O}$ tipo de parto não esteve relacionado com a mortalidade no período neonatal, fato também constatado em um estudo18 que avaliou por 10 anos a redução da mortalidade perinatal e a manutenção das taxas de parto cesárea. No entanto, a cesariana pode apresentar efeito protetor sobre a mortalidade neona- 
tal, principalmente devido sua maior concentração no Brasil em hospitais privados, cuja população de melhor nível socioeconômico detém outras características favoráveis à sobrevivência no período neonatal.12

A ausência de assistência pré-natal associou-se a um maior risco de óbito no período neonatal na análise bivariada, fato também evidenciado em estudos no Sul do México ${ }^{19}$ e do Brasil. ${ }^{20}$ A importância do controle pré-natal nos resultados da gravidez é relacionada a seus amplos objetivos, em que, através de um acompanhamento rigoroso, consegue-se identificar as situações de risco de forma precoce e tomar as devidas providencias. ${ }^{21}$ Mediante as possibilidades do pré-natal é fundamental que as gestantes tenham essa assistência garantida.

Verificou-se, no presente estudo, uma tendência de diminuição da mortalidade neonatal à medida que aumenta o grau de instrução materna, situação já evidenciada em São Paulo10. Na análise multivariada o grau de instrução materna não se associou à mortalidade neonatal. Mesmo que a baixa escolaridade não interfira diretamente na mortalidade neonatal, ela geralmente se relaciona a baixo nível socioeconômico, situação adversa à saúde infantil e materna7 .

As idades maternas consideradas como extremas para a reprodução, menos de 20 e mais de 34 anos, não se constituíram em fatores de risco para a mortalidade nas primeiras semanas de vida, concordando com os achados do Grupo Colaborativo de Estudos Perinatais. 8 Porém, um estudo no Sul do Brasil7 detectou resultados discordantes, onde a idade materna superior a 35 anos foi uma condição que aumentou em cinco vezes o risco de óbitos neonatais precoces. Já no município do Rio de Janeiro, a proporção de mães adolescentes por bairros foi a variável mais explicativa na análise espacial da mortalidade neonatal precoce. Isso se justificou pela expressiva correlação entre a elevada proporção de mães adolescentes, a falta de atenção pré-natal, baixo nível de instrução materna e pobreza. $22 \mathrm{O}$ ideal é que a gravidez ocorra de forma planejada em um momento de condições pessoais e assistenciais adequada para uma boa gestação e nascimento.

Pode-se afirmar que os determinantes da mortalidade neonatal na cidade de Montes Claros, foram similares aos resultados encontrados em estudos do mesmo gênero em outras localidades.7,9,10,12,22 Quanto menor o escore de Apgar no primeiro e quinto minutos de vida, menores foram as chances de sobrevivência, concordando com o estudos realizados em São Paulo8,9,23. Sendo o escore de Apgar um indicador da vitalidade, que quando baixo se relaciona à maior letalidade no período neonatal, 9 é importante a adoção de medidas para o suporte adequado às crianças que nascem nessa condição.

$\mathrm{O}$ peso ao nascer inferior a $2.500 \mathrm{~g}$ esteve fortemente relacionado aos óbitos do período neonatal, mesmo controlando todas as outras variáveis. O BPN é isoladamente o fator que mais afeta a mortalidade neonatal, o que é comprovado por vários autores em diferentes épocas e países. 9,11,24,25,26 O BPN se associa a nascimentos prematuros e/ou retardo do crescimento intra-uterino, situações decorrentes de problemas de saúde materna, destacando-se a hipertensão arterial, de assistência ao pré-natal e condições econômicas desfavoráveis. 6

A idade gestacional inferior a 37 semanas foi a variável que apresentou maior força de associação com a mortalidade no período neonatal. A prematuridade como um dos principais fatores de risco para a mortalidade neonatal é consenso na literatura, bem como a associação entre prematuridade e baixo peso ao nascer.6,7,12 Esse achado aponta para a necessidade de disponibilidade de recursos tecnológicos e humanos adequados para o atendimento em tais circunstâncias. ${ }^{27}$ A falta de unidades de terapias intensivas na cidade de Montes Claros, na época deste estudo, pode ter dificultado a prevenção de óbitos potencialmente evitáveis entre os prematuros.

Os fatores relacionados à mortalidade neonatal encontrados no presente estudo foram essencialmente biológicos, mas passíveis de influências assistenciais e socioeconômicas capazes de interferir nesses determinantes. Portanto, as ações para alterar essa realidade devem acontecer de forma ampla, envolvendo várias esferas dos serviços de saúde e da sociedade com o objetivo primeiro de evitar o nascimento em situação de risco, e quando mesmo assim esse ocorrer, oferecer um suporte adequado visando à sobrevivência sem sequielas danosas. Faz-se necessário o fortalecimento dos sistemas de atendimento obstétrico e neonatal através dos serviços hospitalares de alta complexidade, além da utilização de tecnologias básicas preventivas na assistência pré-natal.

Nesse sentido, o Ministério da Saúde do Brasil apresentou as principais diretrizes para reorganização da rede de assistência à infância nos seus vários níveis, tendo como eixos fundamentais para a redução da mortalidade neonatal a promoção do nascimento saudável e o acompanhamento do recémnascido de risco. ${ }^{3}$

Portanto, esforços devem ser empreendidos para garantir os serviços de comprovada eficácia na redução das ocorrências dos determinantes dos óbitos neonatais, visto que se torna cada vez menos admissível que a morte continue ocorrendo quando existe tecnologia para sua prevenção. 


\section{Agradecimentos}

À Fundação de Amparo à Pesquisa do Estado de Minas Gerais pela inclusão no Programa de capacitação de Recursos Humanos (PCRH) e a Wálter Ferraz pela elaboração do programa informatizado para cruzamento SIM-SINASC.

\section{Referências}

1. Victoria GC, Barros FC. Infant mortality due to perinatal causes in Brazil: trends, regional patterns and possible interventions. São Paulo Med J 2001; 119: 33-42.

2. Leal MC, Szwarcwald CL. Evolução da mortalidade neonatal no Estado do Rio de Janeiro, Brasil, de 1979 a 1993. 1- Análise por grupo etário segundo região de residência. Rev Saúde Pública 1996; 30: 403-12.

3. Ministério da Saúde. Agenda de Compromissos para a Saúde Integral da Criança e Redução da Mortalidade Infantil. Brasília (DF): O Ministério; 2004.

4. Leal MC, Szwarcwald CL. Características da mortalidade neonatal no Estado do Rio de Janeiro na década de 80: uma visão espaço-temporal. Rev Saúde Pública 1997; 31: 457-65.

5. Ministério da Saúde. Manual de atenção ao recém-nascido. Brasília (DF): O Ministério; 1994.

6. Marcondes E. Pediatria básica. São Paulo: Sarvier; 1994.

7. Araújo BF, Bozzetti MC, Tanaka ACA. Mortalidade neonatal no município de Caxias de Sul: um estudo de coorte. J Pediatr [Rio de J] 2000; 76: 200-6.

8. GCEP(Grupo Colaborativo de Estudos Perinatais). Fatores perinatais relacionados com a morbidade e a mortalidade de recém-nascidos pertencentes a nove unidades neonatais do município de São Paulo. J Pediatr [Rio de J] 1996; 72: 379-87.

9. Coutinho SB. Mortalidade neonatal em cinco maternidades da cidade de Recife 1994: relatório de pesquisa. Recife: UNICEF; 1996.

10. Ortiz LP. Características da mortalidade neonatal no Estado de São Paulo [tese doutorado]. São Paulo: Faculdade de Saúde Pública Universidade de São Paulo; 1999.

11. Sarinho WS, Djalma AMF, Silva GAP, Lima MC Fatores de risco para óbitos neonatais no Recife: um estudo caso-controle. J Pediatr [Rio de J] 2001; 77: 294-8.

12. Morais Neto OL, Barros MBA. Fatores de risco para a mortalidade neonatal e pós-neonatal na região CentroOeste do Brasil: linkage entre bancos de dados de nascidos vivos e óbitos infantis. Cad Saúde Pública 2000; 16 : 477-85.

13. Bohland AK, Mello Jorge MHP. Mortalidade de menores de um ano de idade na região Sudeste do estado de São Paulo. Rev Saúde Pública 1999; 33: 366-73.

14. Theme Filha MM, Gama SGN, Cunha CB, Leal MC. Confiabilidade do Sistema de Informações sobre Nascidos Vivos Hospitalares no município do Rio de Janeiro

1999-2001. Cad Saúde Pública 2004; 20 [Supl 1]: p. 8391.

15. Ministério da Saúde. Mortalidade neonatal no Brasil e no Estado de Minas Gerais: 1997-1999. Disponível em: http://tabnet.datasus.gov.br/cgi/idb2002/c0102.htm. [2004 set 28].

16. Yu V. Global, regional and national perinatal and neonatal mortality. J Perinat Med 2003; 31: 376-9.

17. Victoria GC, Barros FC, Vaughan JP. Epidemiologia da desigualdade: um estudo longitudinal de 6.000 crianças brasileiras. São Paulo: Hucitec; 1988.

18. Duarte G, Coltro PS, Bedone RV, Nogueira AA, Gelonezzi GM, Franco LJ. Trends in the modes of delivery and their impact on perinatal mortality rates. Rev Saúde Pública 2004; 38: 379-84.

19. Montero-Mendonza E, Salvatierra-Izaba B, NazarBeutelspacher A. Efectos del tipo de atención del parto em la mortalidad perinatal em Tapachula, Chiapas, frontera sur de México. Atención Primaria 2000; 25: 56874.

20. Menezes AMB, Barros FC, Victora CG, Alves C, Rocha C, Albernaz E, Menezes F, Jannke HA. Mortalidade perinatal em duas coortes de base populacional no sul do Brasil: tendências e difereciais. Cad Saúde Pública 1996; 12 [Supl 1]: 33-41.

21. De Lorenzi DRS, Tanaka ACD'A, Bozzettim C, Ribas FE, Weissheimer L. A natimortalidade como indicador de saúde perinatal. Cad Súde Pública 2001; 17: 141-6.

22 Andrade CLT, Szwarcwald CL. Análise espacial da mortalidade neonatal precoce no Município do Rio de Janeiro, 1995-1996. Cad Saúde Pública 2001; 17: 1199-210.

23. Thorngren-Jerneck K, Herbest A Low 5-minute Apgar score: a population-based register study of 1 million term births. Obstet Gynecol 2001; 98: 65-70.

24. Puffer RR, Serrano CV. Utilização dos dados hospitalares de peso ao nascer e mortalidade como indicadores de problemas de saúde na infância. In: OPAS (Organização Panamericana da Saúde), OMS (Organização Mundial da Saúde). Investigação de mortalidade na infância no Brasil: descobertas e atividades. Washington (DC): As Organizações; 1977. p. 62-87. (Publicação científica, 343).

25. Acciolly MC. Determinantes da mortalidade neoonatal em Belo Horizonte (1993): subsídios para o planejamento da atenção materno-infantil [dissertação mestrado]. 
Belo Horizonte: Faculdade de Medicina Universidade Federal de Minas Gerais Belo Horizonte; 1997.

26. Kochanek KD, Martin JA. Supplemental analyses of recent trends in infant mortality. National Center for Health Statistics. Disponível em: http://www.cdc.gov.nchs. products/pubs/pubd/hestats/infantmort/infantmort.htm. [2004 out 23].

27. Alexander GR, Kogan M, Bader D, Carlo W, Allen M, Mor J. US birth weight/gestational age-specific neonatal mortality: 1995-1997 rates for whites, hispanics, and blacks. Pediatrics 2003; 111: 61-6.

Recebido em 1 de outubro de 2003

Versão final apresentada em 28 de setembro de 2004

Aprovado em 1de outubro de 2004 